\title{
Avaliação neuropsicológica das funções executivas de mulheres em estado de dependência química
}

\author{
Neuropsychological assessment of the executive functions of women \\ in a state of chemical addiction
}

\section{La evaluación neuropsicológica de las funciones ejecutivas de las mujeres en un estado de dependência química}

\author{
Edilson Ramiro FREITAS \\ Rui Mateus JOAQUIM² \\ Maria de Lourdes Merighi TABAQUIM ${ }^{3}$ \\ Ana Paula CAMARGO \\ ${ }^{1}$ Psicológo, Graduado pela Faculdade Anhanguera de Bauru \\ ${ }^{2}$ Doutorando do Laboratório de Neuropsicologia do Hospital de Reabilitação de Anomalias Craniofaciais da \\ Universidade de São Paulo. (HRAC/USP) \\ ${ }^{3}$ Neuropsicóloga, Docente do Departamento de Fonoaudiologia da Faculdade de Odontologia de Bauru da \\ Universidade de São Paulo(FOB/USP) e do Hospital de Reabilitação de Anomalias Craniofaciais da Universidade de São Paulo(HRAC/USP) \\ ${ }^{4}$ Doutoranda do Departamento de Neurociência e Comportamento da Faculdade de Medicina da Universidade de São Paulo. (FMRP/USP)
}

\section{Resumo}

Introdução: O funcionamento executivo preservado para a manutenção de comportamentos adaptativos é condição necessária para obtenção de desfechos clínicos favoráveis no tratamento de sujeitos em dependência química. A confirmação da hipótese de disfunção executiva pode fornecer subsídios ao tratamento comportamental, do sujeito com dependência química. Objetivo: O estudo consistiu na realização de uma avaliação neuropsicológica das funções executivas de mulheres dependentes químicas de cocaína ou crack. Método: A avaliação se deu através de anamnese/exame clínico, entrevistas e testes neuropsicológicos. Para a caracterização da amostra foi utilizado o Protocolo de Anamnese Neuropsicológica. A avaliação neuropsicológica das funções executivas consistiu da aplicação do Wisconsin Card Sorting Test (WCST), Subteste Dígitos - Wechsler Adult Intelligence Scale (WAIS-III), Blocos de Corsi, Trail Making Test (TMT), Stroop Test e o Montreal Cognitive Assessment (MoCA), a fim de investigar oito componentes executivos, a saber: memória operacional, flexibilidade cognitiva, categorização, fluência verbal, atenção seletiva e alternada, rastreamento visuomotor e controle inibitório. Resultados: Os resultados permitiram concluir que mulheres com dependência química, pelo uso de cocaína e crack, apresentam disfunção executiva. Foram encontradas relações clinicamente significativas entre tempo de uso e déficits do funcionamento executivo, indicando que quanto maior o tempo de dependência, mais expressiva a disfunção executiva.

Descritores: Função Executiva; Neuropsicologia; Transtornos Relacionados ao Uso de Substâncias.

\begin{abstract}
Introduction: Executive functioning preserved to maintain adaptive behavior is a prerequisite for obtaining favorable clinical outcomes in the treatment of subjects in addiction. Confirmation of the executive dysfunction hypothesis can provide subsidies to behavioral treatment of the subject with addiction. Objective: The study consisted of a neuropsychological assessment of executive functions of addicted women cocaine or crack. Method: The evaluation was made through medical history / physical examination, interviews and neuropsychological tests. For the characterization of the sample was used Anamnese Neuropsychological Protocol. Neuropsychological assessment of executive functions consisted of applying the Wisconsin Card Sorting Test (WCST), subtest Digit - Wechsler Adult Intelligence Scale (WAIS-III), Corsi blocks, Trail Making Test (TMT), Stroop Test and the Montreal Cognitive Assessment ( MoCA) in order to investigate eight executive components, namely: working memory, cognitive flexibility, categorization, verbal fluency, selective and alternating attention, visuomotor tracking and inhibitory control. Results: The results showed that women with substance abuse, the use of cocaine and crack, present executive dysfunction were found clinically significant relationship between use of time and deficits in executive functioning, indicating that the higher the dependence on time, more significant dysfunction executive.

Descriptors: Executive Function; Neuropsychology, Temporary; Substance-Related Disorders.
\end{abstract}

\begin{abstract}
Resumen
Introducción: El funcionamiento ejecutivo conservado para mantener la conducta adaptativa es una condición necesaria para la obtención de los resultados clínicos favorables en el tratamiento de sujetos en la adicción. La confirmación de la hipótesis de la disfunción ejecutiva puede proporcionar subsidios a tratamiento conductual del sujeto con dependencia química. Objetivo: El estudio consistió en una evaluación neuropsicológica de las funciones ejecutivas de las mujeres dependientes de cocaína o crack. Método: La evaluación se realiza a través de la historia médica / examen físico, entrevistas y pruebas neuropsicológicas. Para la caracterización de la muestra se utilizó Protocolo del Anamnese Neuropsicológica. La evaluación neuropsicológica de las funciones ejecutivas consistió en aplicar el Wisconsin Card Sorting Test (WCST), subprueba dígito - Escala de Inteligencia de Wechsler para adultos (WAIS-III), Corsi bloques, Trail Making Test (TMT), prueba de Stroop y la Evaluación Cognitiva Montreal ( MOCA) con el fin de investigar los ocho componentes ejecutivos, a saber: la memoria de trabajo, la flexibilidad cognitiva, categorización, la fluidez verbal, atención selectiva y alterna, seguimiento y control inhibitorio visuomotor. Resultados: Los resultados mostraron que las mujeres con el abuso de sustancias, el uso de la cocaína y el crack presentan la disfunción ejecutiva. Fueran encontradas relaciones clínicas significativas entre el uso del tiempo y los déficits en el funcionamiento ejecutivo, lo que indica que cuanto mayor es la dependencia de tiempo, la disfunción ejecutiva es más significativo.
\end{abstract}

Descriptores: Función Ejecutiva; Neuropsicología; Trastornos Relacionados con Sustancias. 


\section{INTRODUÇÃO}

Classificada pela CID- $10^{1}$ como um forte desejo ou compulsão para utilização de drogas, o sujeito com Síndrome de Dependência, encontra muitas dificuldades em controlar o uso de tais substâncias, uma vez que o organismo encontra-se em dependência fisiológica e psíquica. Dada a interrupção ou a redução desse consumo, o sujeito em dependência química evolui para o quadro denominado Síndrome de Abstinência, cujo qual, de acordo com o DSM-IV-TR ${ }^{2}$ pode apresentar os seguintes sintomas: hipersonia; aumento do apetite; sensação geral de infelicidade; estado emocional caracterizado por uma ou muitas emoções consideradas negativas, tais como, tristeza, ansiedade, irritabilidade e inquietação; sintomas psicóticos e desejo intenso pela droga. Deste modo, a experiência desses sintomas certamente vulnerabiliza o indivíduo à continuidade do uso da droga.

Como a CID-10 ${ }^{1}$ descreve, existe o efeito tolerância, configurado por um aumento da dosagem de droga para obtenção dos mesmos efeitos e sintomas, em contraponto, não havendo este aumento, há uma diminuição ou nenhuma sensação. Neste ínterim, o dependente abandona progressivamente prazeres e interesses, com um aumento de atividades para obtenção e utilização da droga. O sujeito, mesmo com a evidência das consequências nocivas do uso da substância, não se abstém dela, atestando deste modo o conceito de dependência química.

Dos componentes químicos altamente viciantes, a cocaína é uma substância que produzida por planta chamada coca, que pode ser consumida de por diferentes maneiras, tais como: ingestão de suas folhas, por mastigação de; a pasta de coca, que pode ser injetada; inalação do hidrocloreto de cocaína; e por uso de alcaloides de cocaína como a freebase e o crack, cujos quais são fumados. Estas várias formas diferem em potência devido às variações dos níveis de pureza e rapidez de início dos respectivos efeitos. A cocaína é o princípio ativo de todas estas preparações. As folhas de coca são mascadas geralmente pelas populações nativas da América Central e do Sul, onde encontra-se estas plantas. A utilização da pasta de coca, que é um extrato puro da planta, é feita nos países produtores da América Central e do Sul. O hidrocloreto de cocaína, o pó, é habitualmente aspirado através das narinas ou dissolvido em água e injetado na via intravenosa ${ }^{2}$.

O crack, por sua vez, é obtido de uma mistura da cocaína com bicarbonato de sódio e, quando secado, transformado em pequenas pedras. Ele difere das outras formas porque é facilmente vaporizado e inalado e, assim, os seus efeitos tem um início extremamente rápido ${ }^{2}$.

Segundo Negrete ${ }^{3}$ o consumo da cocaína pela via pulmonar não era realizado na América do Sul antes dos anos 70. Maass et al. ${ }^{4}$ relatam que o hábito de fumar a pasta de folhas de coca começou a ganhar popularidade, com o aumento progressivo ao longo desta década, tanto nos países produtores, quanto nos Estados Unidos. A pasta de folha de coca ou pasta básica é obtida por meio de solventes (álcool, benzina, parafina ou querosene), ácido sulfúrico e carbonato de sódio.

Conforme Castaño ${ }^{5}$, nos anos 80 surgiu nos Estados Unidos a cocaína na forma de base livre ou freebasing, sintetizada a partir da adição de éter sulfúrico à cocaína refinada, em meio aquoso altamente aquecido.

Reinarman e Levine $^{6}$ revelam que o crack surgiu alguns anos à frente, na década de 80 , entre os anos 84 e 85, nos bairros carentes de Los Angeles, Nova York e Miami. Segundo Ellenborn et al. ${ }^{7}$, os cristais eram fumados em cachimbos e estralavam (craching) quando expostos ao fogo, característica que the conferiu o nome "crack". De acordo com Osap ${ }^{8}$, a utilização desta substância produzia uma euforia de grande magnitude e de curta duração, seguida de intensa fissura e desejo de repetir a dose.

Como afirma Procópio ${ }^{9}$, há poucos relatos de como o crack chegou ao Brasil, as informações são provenientes da imprensa ou órgãos policiais. As apreensões de crack realizadas pela Polícia Federal iniciaram a partir dos anos 90, aumentando 166 vezes no período de 1993-1997, enquanto que a apreensão da pasta básica, no mesmo período na região sudeste aumentou 5,2 vezes.

Segundo Uchôa ${ }^{10}$ algumas evidências apontam para o surgimento da substância em bairros da zona leste como São Mateus, Cidade Tiradentes e Itaim Paulista, e posteriormente na região da Estação da Luz, conhecida como "Cracolândia", no centro da cidade. A partir daí espalhou-se para vários pontos da Capital Paulista, eliciado pelo ambiente de exclusão social e segundo, Dimenstein ${ }^{11}$ pela repressão policial mais forte no centro da cidade.

Nappo et al. ${ }^{12}$ afirmam que a primeira pesquisa sobre o consumo de crack no Brasil foi um estudo etnográfico realizado no município de São Paulo, com 25 usuários vivendo na comunidade. Segundo Dunn e Laranjeira $^{13}$ o aparecimento da substância e a popularização do consumo tiveram início a partir de 1989. O perfil dos sujeitos pesquisados apresentava pessoas do sexo masculino, com idade abaixo de 30 anos, desempregados, com baixo nível de escolaridade e poder aquisitivo, procedentes de famílias desestruturadas.

Dados apresentados por Nappo et al. ${ }^{12}$ indicaram que o consumo era eliciado pela compulsão, dependência ou como mecanismo de enfrentamento desadaptativo para lidar com problemas familiares e 
frustrações. A fixação dos usuários pelo consumo de crack sobrepunha necessidades de sono, alimento, comportamento afetivo e senso de responsabilidade.

Dias et al. $^{14}$ e Abeid-Ribeiro ${ }^{15}$ revelam que quanto ao tempo de uso, existe um grupo de usuários que utiliza o crack há mais de dez anos de forma ininterrupta, com a probabilidade para uma adaptação do usuário à cultura do uso.

Quanto à epidemiologia, de acordo com dados recentes aferidos através do II Levantamento Nacional de Álcool e Drogas ${ }^{16}$ desenvolvido pela Universidade Federal de São Paulo (UNIFESP), o Brasil é o segundo maior consumidor de cocaína e derivados, atrás apenas dos Estados Unidos. Esta pesquisa, realizada em 149 municípios, das cinco regiões do país, se deu através de 800 perguntas a 4.607 pessoas com idade mínima de 14 anos, sobre o consumo de cocaína aspirada ou fumada como também de outras drogas. O estudo mostra que o Brasil responde hoje por $20 \%$ do mercado mundial da droga. Sobre a incidência da cocaína por regiões, se evidenciou que o consumo é três vezes nas áreas urbanas, principalmente no Sudeste, com $46 \%$ dos usuários, ou 1,4 milhões de pessoas. Posteriormente o Nordeste (27\%), o Norte e o Centro-oeste (10\%, cada) e o Sul (7\%). Este tipo de problemática tem exigido que novas linhas de investigação sejam encontradas, tendo por objetivo a busca de solução, dada os expressivos índices apresentados.

\section{CÉREBRO E COGNIÇÃo}

O crescente interesse pelo funcionamento cerebral e, por consequência, cognitivo, tem suas bases arraigadas na antiguidade, maturadas ao longo dos tempos, uma vez que técnicas foram sendo aprimoradas, para que se chegasse aos pressupostos atuais.

Em meados de 1970, psicólogos cognitivistas não tinham ainda um entrosamento com a neuropsicologia, mas não demorou, para que a psicologia cognitiva unisse seu modelo à investigação da organização funcional das habilidades cognitivas, por meio da elaboração de testes em indivíduos normais sem um caráter clínico, embora toda a história de observações estivesse impregnada de estudos de sujeitos com alguma patologia ${ }^{17}$. Talvez, por ser o resultado da convergência de várias ciências, a neuropsicologia tardou em oferecer formação direcionada aos profissionais interessados, bem como em reunir e organizar um corpo teórico específico.

A construção de conceitos imprescindíveis à prática clínica foi enriquecida a partir do desenvolvimento de pesquisas cientificas. Seguindo o pressuposto de que processos psicológicos podem ser investigados por exames não invasivos, tais como: testes, inventários e questionários (procedimentos padronizados e capazes de descrever, com certa fidedignidade como capacidades e habilidades mentais se comportam após algum tipo de lesão cerebral), ou mesmo assessorando estudos comparativos transculturais ${ }^{17}$.

Por outro lado, ao longo dos anos, a neuropsicologia esteve estruturada sobre o estudo de casos únicos, dependente de uma observação cuidadosa do comportamento exibido pelo paciente, e, muitas vezes guiada pelas estruturas provenientes da psicologia cognitiva. Nos anos 80, tornou-se insustentável que neuropsicologia e psicologia cognitiva se mantivessem distantes e alheias. O encontro entre estas duas áreas disponibilizou que se abrisse um canal de comunicação, como decorrência surgiu eventos, publicações e pesquisas em conjunto. Desde então a parceria denominada neuropsicologia cognitiva tem incrementado a demanda de produções a partir das trocas de informações, material teórico e experiência clínica.

Sem perder o perfil tradicional, a neuropsicologia se mantém estudando a localização e organização funcional, bem como a ação dinâmica de seus componentes. Enquanto, a psicologia cognitiva, mais do que o nível de análise teórica, ganhou maior clareza e agilidade na comprovação de suas hipóteses. Portanto, a formação de grupos interdisciplinares parece ser uma tendência viável para a ciência do novo milênio. Tendo por exemplo, as neurociências, que englobam: o estudo da neuroanatomia; neurofisiologia; neuroquímica e as ciências do comportamento $^{17}$.

Segundo Beatty ${ }^{18}$, atualmente os neurocientistas atuam de forma sincronizada e harmoniosa, sendo monitorados todos os resultados entre estas áreas. Esta mudança de atitude tem gerado maior rapidez e aumento do conhecimento sobre o cérebro e do controle que ele exerce sobre o comportamento.

Estudos realizados por Cosenza e Guerra ${ }^{19} \mathrm{e}$ Fuentes et al. ${ }^{20}$ apontam a neuropsicologia como um campo do conhecimento que estabelece as relações existentes entre o funcionamento do sistema nervoso central e o comportamento, nas condições normais e patológicas. Sua natureza é multidisciplinar, com fundamentos das neurociências e da psicologia, visando ao tratamento dos distúrbios cognitivos e comportamentais, decorrentes de alterações no funcionamento do sistema nervoso central.

\section{FUNÇÕES EXECUTIVAS E DEPENDÊNCIA QUÍMICA}

Conforme Esteves-Gonzáles et al. ${ }^{21}$ a partir da observação de pessoas com lesões frontais e alterações comportamentais subsequentes a estas lesões, surgiu as primeiras ideias de avaliar as funções deste sistema. No século XIX houve vários casos como os frenologistas Gall ${ }^{22}$ e Spurzheimer ${ }^{23}$ e suspeitavam 
que os lobos frontais fossem responsáveis pela fala e cálculo.

Broca apud Freinberg e Farah $^{24}$ descreveu diversos casos de afasia com lesões nos lobo frontal esquerdo, bem como Harlow apud Damásio ${ }^{25}$ com o famoso caso de Phineas Gage com suas alterações comportamentais. Com Luria ${ }^{26}$, por sua vez, a avaliação neuropsicológica dos lobos frontais começou a ser estruturada por meio de estudos com pacientes lesionados na Segunda Guerra Mundial, delineando-se um modelo para as lesões nos lobos frontais, o qual centraliza as responsabilidades por planejamentos, programação, regulação e verificação do comportamento intencional.

Entretanto, foi Lezak (1995), quem deu o nome Funções Executivas ao termo, dividindo-as nas seguintes categorias: a formulação de metas, planejamento, realização de planos dirigidos às metas e execução efetivas de atividades dirigidas as metas. Para Cosensa e Guerra $^{19}$, embora não haja um consenso sobre a conceituação das funções executivas, é possível defini-las como o conjunto de habilidades e capacidades que nos permitem executar as ações necessárias para atingir um objetivo. Nelas estão incluídos: a identificação de metas, o planejamento de comportamentos e sua execução, além do monitoramento do próprio desempenho, até que o objetivo seja consumado. Elas devem assegurar, além disso, que as normas sociais sejam respeitadas, em um padrão comportamental considerado apropriado para um determinado contexto ou situação.

As funções executivas possibilitam nossa interação com o mundo frente às mais diversas situações que encontramos. Por meio delas organizamos nosso pensamento, levando em conta as experiências e conhecimentos armazenados em nossa memória, assim como nossas expectativas em relação ao futuro, sempre respeitando os valores e propósitos individuais. Dessa forma, podemos estabelecer estratégias comportamentais e dirigir nossas ações de uma maneira mais objetiva, mas flexível que permita, ao final, chegar ao objetivo desejado. Além disso, são as funções executivas que suportam uma supervisão de todo o processo, evitando erros e limitando nossas ações dentro dos padrões éticos do grupo cultural a que pertencemos ${ }^{19}$.

Segundo Figlie et al. $^{27}$ as substâncias psicoativas presentes na cocaína atuam diretamente no Sistema de Recompensa Cerebral composto pelo córtex pré-frontal, nucleus accumbens, amígdala e área tegmentar ventral. O Sistema de Recompensa Cerebral pode ser chamado também de centro de prazer. Atos como comer, tocar um instrumento musical ou ter relações sexuais proporcionam ao indivíduo prazer. Deste modo, sendo altamente reforçadores, a tendência do comportamento é a manutenção deles.

A dopamina, por sua vez, é o principal neurotransmissor atuante no circuito de recompensa. Neste ínterim, ocorre que, com o uso da cocaína, o funcionamento neuronal altera-se totalmente, visto que essa substância inibe a recaptura da dopamina pelo neurônio pré-sináptico. Frente a este efeito, ocorre um excesso neuroquímico na fenda sináptica, acarretando uma sensação de prazer, consequentemente, causando uma recompensa não natural e alterando o $\mathrm{SRC}^{27}$.

De acordo com $\mathrm{Cunha}^{28}$, no decorrer do tempo, esse circuito começa a depender da droga para executar suas funções normalmente, produzindo menos dopamina, e gerando ansiedade, humor alterado, anedonia, diminuição de energia e até mesmo déficits cognitivos.

De acordo com Nassif ${ }^{29}$, as pesquisas sobre os prejuízos causados pelo uso crônico da cocaína, surgiram nos anos 90. Essa década foi denominada a "Década do Cérebro", e a contribuição da neuroimagem possibilitou acréscimos significativos nos conhecimentos, sobre as alterações neurológicas produzidas por esta substância psicoativa. Com isto, cada vez mais foram sendo estimuladas pesquisas de avaliação de déficits cognitivos associados ao seu uso crônico.

Segundo Verdejo et al. ${ }^{30}$ o acometimento das funções cognitivas que podem estar afetadas pelo efeito neurotóxico acumulativo do consumo de drogas sobre diversos mecanismos, pode justificar alguns tipos de conduta do usuário de crack. Existem três aspectos anormais de conduta comumente presentes em dependentes químicos e tradicionalmente relacionados com os lobos frontais: (1) componente de expectativa (recompensa); (2) componente compulsivo; e, (3) componente de tomada de decisões.

Conforme Lorea et al. ${ }^{31}$, os prejuízos na fluidez verbal também trazem repercussões significativas na conduta do dependente químico. Uma diminuição nas estratégias de busca pode limitar durante um tempo indeterminado sua capacidade de encontrar novas atividades, ou uso de tempo, durante a reabilitação. Afirmam também que há uma dificuldade sobre o controle inibitório, sendo que uma das condutas de base observadas em usuários desta substância é a perda de controle sobre a própria conduta. E afirmam que é evidente o comprometimento neuropsicológico de várias funções cognitivas, principalmente da memória, da aprendizagem e das funções executivas.

Nassif ${ }^{29}$ e Verdejo et al. $^{31}$ afirmam que com o uso mais prolongado da droga, começa a surgir também perdas na capacidade de tomada de decisão, o que se dá pelo acometimento mais significativo dos 
lobos frontais, assim como perdas quanto ao controle inibitório, atenção, memória operacional, memória verbal, raciocínio, funções abstratas e funções psicomotoras. Do mais, os estudos demonstraram que a utilização do crack está correlata a prejuízos mais velozes, especialmente, de funções como: memória verbal, atenção e funções executivas.

Bolla et al. ${ }^{32}$ afirmam que o uso prolongado de cocaína pode causar anormalidades no funcionamento do córtex pré-frontal persistentes, que interferem no processo de tomada de decisão. Uma paciente que tem dificuldades em tomar decisões ficará vulnerável às decisões sem uma pré-análise ou ficará muito indecisa, o que causará insegurança e vulnerabilidades, com isto, poderá retornar ao uso da substância, com consequente abandono de tratamento.

Estudos realizados com dependentes de cocaína revelam um baixo desempenho em testes que avaliam: atenção, funções executivas, memória visual de longa duração, aprendizagem verbal, memória de trabalho, resolução de problemas e tomada de decisão, quando comparados a grupo controle ${ }^{17,28}$.

Dado este contexto, a recuperação de uma dependente química passa por problemas e ela deverá ter a habilidade de resolvê-los, por isso tratamento mais indicados apontam para a utilização de Treino de Habilidades Sociais, cuja eficácia fica significativamente comprometida, quando um déficit cognitivo é correlato. Sendo assim, déficits das funções executivas podem afetar o tratamento, pois os sujeitos podem encontrar dificuldades na compreensão e assimilação de conceitos básicos da terapia, bem como influenciar o traçado de metas e o cumprimento de objetivos que não envolvam uma recompensa imediata, tal qual a inibição de respostas impulsivas inapropriadas $^{28,33}$.

\section{MATERIAL E MÉTODO}

A presente pesquisa trata-se de uma coorte transversal de sujeitos do sexo feminino, em internação para tratamento de dependência química, avaliados durante o período de agosto a outubro, do ano de 2014.

\section{$\checkmark \quad$ Procedimento Ético}

O presente estudo foi submetido à avaliação do Comitê de Ética em Pesquisa, por intermédio do acesso à Plataforma Brasil, tendo sido autorizado, houve a seleção da amostra e os sujeitos que cumpriram os requisitos, dentro da instituição, deram o consentimento formal assinando o Termo de Consentimento Livre e Esclarecido.

\section{$\checkmark$ Caracterização da Amostra}

A amostra do presente estudo foi constituída por pacientes admitidas em uma Comunidade Terapêutica.

\section{$\checkmark$ Critérios de Exclusão do Estudo}

A seleção dos sujeitos ocorreu mediante a utilização dos seguintes critérios de exclusão e inclusão:

1. Sexo masculino;

2. Idade inferior a18 anos;

3. Dependente química de drogas que não seja cocaína ou crack;

4. Analfabetismo.

Sendo aplicados os critérios de exclusão inicial, eram incluídos no estudo sujeitos que consentiam formalmente a participação no estudo.

\section{$\checkmark$ Caracterização Sociodemográfica e Clínicas dos Sujeitos}

A idade média dos sujeitos avaliados foi de 28 anos $(\mathrm{dp}=7,6)$, sendo a idade mínima 19 e a máxima 49 anos, todas do sexo feminino. A média de escolaridade foi de 8,15 anos de estudo $(d p=2,4)$. O período de dias de internação dos sujeitos, quando foram aplicados os instrumentos, foi de 30 a 120 dias (Tabela 1).

Tabela 1. Características sociodemográficas e clínicas dos sujeitos

\begin{tabular}{cccc}
\hline Variável & & & \\
\hline $\begin{array}{c}\text { Variável Numérica } \\
\text { Idade }\end{array}$ & Média & dp & Mediana \\
Dias Internação & 64,45 & 7,6 & 27 \\
Variável Categórica & & 54,5 & 39 \\
Escolaridade Anos & 8,15 & 2,4 & 8 \\
\hline
\end{tabular}

\section{$\checkmark$ Materiais e instrumentos}

A avaliação neuropsicológica dos oito domínios cognitivos correspondentes às funções executivas, a saber: memória operacional, flexibilidade cognitiva, categorização, fluência verbal, atenção seletiva e alternada, rastreamento visuomotor e controle inibitório, foi realizada pelos seguintes testes:

1) Wisconsin Card Sorting Test (WCST) - Teste Wisconsin de Classificação de Cartas $^{34}$ : foi desenvolvido para avaliar a capacidade de raciocínio abstrato e a capacidade para modificar estratégias cognitivas em resposta a mudanças, ou seja, a capacidade para desenvolver e manter uma estratégia apropriada de solução de problemas por meio de condições de estímulos mutáveis, a fim de atingir uma meta futura. O WCST fornece escores objetivos tanto do sucesso total como de dificuldades na execução da tarefa. É constituído por quatro cartas-estímulo e 128 cartas-resposta, que representam quatro formas geométricas (triângulo, estrela, cruz ou círculo), em diferentes cores (vermelho, verde, amarelo ou azul) e números de figuras (um, dois, três ou quatro).

2) Subteste Dígitos-Wechsler Adult Intelligence Scale (WAIS-III) - Escala de Inteligência Wechsler 
para Adultos $^{35}$ : é utilizado para avaliar um componente fluído da memória operacional, denominado alça fonêmica. A tarefa consiste em instruir o sujeito a repetir uma série de dígitos numéricos apresentados pelo examinador, da forma como ditos (ordem direta). Em segundo o examinador dita uma sequência de dígitos e orienta o examinando a evocá-los em ordem inversa.

3) Blocos de Corsi $^{36}$ - o teste oferece um contraponto não verbal à tarefa de dígitos, possibilitando uma medida relacionada ao componente visuoespacial da memória operacional. Em um tablado preto com nove cubos idênticos, distribuídos de maneira uniforme, o sujeito é orientado a repetir sequências de toques realizados pelo pesquisador, primeiramente em ordem direta e posteriormente em ordem inversa.

4) Trail Making Test (TMT) - Formas A e $\mathrm{B}^{37}$ : avalia a capacidade de manutenção do engajamento mental, rastreamento visual, destreza motora, memória operacional, flexibilidade mental e capacidade inibitória. Está dividido em duas partes, A e B. Na parte A, é solicitado ao sujeito que desenhe linhas unindo círculos numerados de forma consecutiva, e, na parte $\mathrm{B}$, sensível à avaliação da flexibilidade cognitiva, solicita-se que o examinando ligue os círculos com números e letras, intercalando-os em ordem crescente.

5) Stroop Test ${ }^{38}$ : divido em três partes, inicialmente, consiste em séries de retângulos coloridos, onde é solicitado ao sujeito a nomeação de cores destes retângulos; posteriormente é solicitado que se nomeie as cores em que as palavras aleatórias estão escritas; e por fim o sujeito deve nomear cores de palavras (cores) escritas, que estão impressas em cores diferentes (incongruência entre a cor da palavra e o que está escrito). Os erros podem ser atribuídos a um conflito de resposta, erro de resposta ou atenção seletiva.

6) Montreal Cognitive Assessment (MoCA) que é um conjunto de 11 testes. Começa com o Trail Making Test (TMT) forma B adaptado. Depois se pede para se desenhar um cubo como está no modelo da folha de aplicação, e logo após para desenhar um relógio com números e ponteiros, sendo que os ponteiros devem indicar onze horas e dez minutos. Estes dois testes analisam as capacidades visuo-espaciais. O próximo é o teste de nomeação, onde são apresentados três animais: Leão, Rinoceronte e Camelo, onde o avaliado deve nomear estes animais. $\mathrm{O}$ seguinte é o de memória, onde será falada cinco palavras: Rosto, Veludo, Igreja, Margarida e Vermelho; e o avaliado deverá repeti-las ao final da sequência, haverá duas chances e logo após será informado, para o avaliado, que guarde na memória e que depois será pedido para ele falar as mesmas palavras. O outro será da atenção, onde será lida uma sequência de cinco dígitos, que deverá ser repetida exatamente na mesma ordem, e depois será lida uma sequência de três dígitos, onde o avaliado deverá repetir em ordem inversa. Logo após o teste de concentração (cancelamento), onde será lida uma sequência de letras e toda vez que se disser a letra A o avaliado deverá dar uma batida na mesa. O próximo será a subtração em sequência de 7 , onde o avaliado deverá começar uma sequência de cinco subtrações, começando com 100. O seguinte será a repetições de duas frases: "Eu somente sei que é o João quem será ajudado hoje." e "O gato sempre se esconde embaixo do sofá quando o cachorro está na sala.". O próximo será da fluência verbal, onde será pedido que o avaliado diga o maior número possível de palavras que comecem com a letra $\mathrm{F}$ dentro de um minuto, exceto nomes próprios de pessoas e lugares; onde se dará o ponto se ele disser no mínimo 11 palavras. O seguinte será o abstração (semelhanças), pede-se ao sujeito que diga o que tem em comum entre dois elementos: "trem e bicicleta" e "relógio e régua", não será qualquer resposta aceita, somente aquelas que constarem na lista do teste. Depois vem a evocação diferida, onde o sujeito deverá lembrar as cinco palavras: "rosto, veludo, Igreja, margarida e vermelho" que foram lidas a pelo menos cinco minutos atrás, pode ser em sequência diferente, onde deve-se dar um ponto a cada resposta certa. E o último é o de orientação, onde o sujeito deve falar que dia é hoje, mês, ano, dia da semana, local onde está e cidade.

Para a análise dos desempenhos obtidos os instrumentos foram categorizados em domínios, conforme a função cognitiva correspondente, de acordo com o Quadro 1.

\begin{tabular}{|cc|}
\hline Instrumentos de Avaliação & Função Executivas \\
Wisconsin Card Sorting Test & Categorização,flexibilidade cognitiva e \\
impulsividade atencional. \\
Digitos (WAIS)- ordem direta e inversa & Memória operacional auditiva \\
Blocos de Corsi & Memória operacional visuoespacial \\
Trail Making Test (A e B) & Atenção seletiva e alternada, rastreamento \\
& visuomotor, rastreamento visuomotor, \\
Stroop Test & Atenção seletiva e controle initório \\
\hline
\end{tabular}

Quadro1. Instrumentos Neuropsicológicos para a avaliação de domínios específicos das funções executivas, adaptado de Camargo ${ }^{39}$

\section{$\checkmark$ Local}

A presente pesquisa foi realizada na Unidade de Psicologia da Comunidade Terapêutica Feminina Bom Pastor.

\section{$\checkmark$ Procedimento de coleta de dados}

Foi apresentada uma autorização para coletas de dados para as pacientes da Comunidade Terapêutica, conforme a Resolução do Conselho Nacional de Saúde CNS-196/96 e assinaram o Termo de Consentimento Livre e Esclarecido, onde as pacientes tiveram a liberdade de escolha à participação espontânea no estudo. A seguir, as pacientes foram submetidas às 
seguintes avaliações: Protocolo de Anamnese Neuropsicológica e Protocolo de Avaliação Neuropsicológica, que corresponde ao Wisconsin Card Sorting Test, Subteste Dígitos - Wechsler Adult Intelligence Scale (WAIS-III), Blocos de Corsi, Trail Making Test, Stroop Test e o Montreal Cognitive Assessment (MoCA).

Durante a avalição, os sujeitos foram submetidos aos instrumentos, de forma individual, conforme critérios normativos de aplicação, para posterior avaliação dos resultados.

\section{$\checkmark$ Procedimento de análise de dados}

Os dados desta pesquisa foram organizados descritivamente, em tabelas e gráficos. Para as variáveis escalares, a análise foi feita por meio da observação dos valores mínimos e máximos, e a partir da obtenção de médias e desvios padrões. Para as variáveis categóricas, calcularam-se as frequências absolutas e porcentagens.

Os resultados brutos, obtidos na avaliação neuropsicológica, foram transformados em percentis, de acordo com as normas dos instrumentos e classificados pelo grau de desempenho elucidado, conforme Spreen e Strauss ${ }^{40}$. Embora cada instrumento tenha o seu perfil de resultados, utilizouse a classificação de percentis para padronizar os níveis de comparação.

A análise estatística foi realizada por meio do programa "Minitab Statistical Software" (MINITAB), versão 16.0, e considerado o nível alfa de 0,05, relativo à significância. Para a análise de correlação entre as variáveis do estudo, foi utilizado o Teste de Correlação de Spearman. A escolha de um teste não paramétrico deu-se em função do tamanho amostral e distribuição anormal dos resultados.

\section{RESULTADOS}

Os resultados do presente estudo foram organizados em tabelas e gráficos e os dados submetidos ao estudo estatístico de cunho descritivo e inferencial. Em função da distribuição anormal dos resultados de testes neuropsicológicos para uma mesma medida, foram atribuídas medianas para análise descritiva.

Quando analisado o desempenho cognitivo global das funções executivas, correspondentes a: categorização; flexibilidade cognitiva; controle inibitório; memória operacional auditiva e visuoespacial; atenção seletiva, alternada; os resultados indicaram uma mediana equivalente a 8 $(\mathrm{dp}=19,56)$, o que caracteriza desempenho inferior, para as medidas de funcionamento executivo.

Em função da significativa variabilidade de desempenhos em diferentes testes neuropsicológicos, a Tabela 2 reflete os resultados estratificados por teste cognitivo e demonstra prejuízo cognitivo em $62,5 \%$ dos instrumentos selecionados neste estudo.

Tabela 2. Desempenho cognitivo da amostra estratificado por teste neuropsicológico

\begin{tabular}{|c|c|c|c|}
\hline Testes Neuropsicológicos & Média $(d p)$ & Mediana & Classificação \\
\hline Corsi- bloco span & $22,1(22,2)$ & 11,50 & $\begin{array}{l}\text { Médio } \\
\text { inferior }\end{array}$ \\
\hline Corsi - pontuação total & $3,65(1,11)$ & 1,00 & Muito inferior \\
\hline Stroop 1 - tempo & $0,85(2,13)$ & 0,00 & Muito inferior \\
\hline Stroop 1 - erros & $53,10(26,47)$ & 66,00 & Médio \\
\hline Stroop 2 - tempo & $0,75(1,91)$ & 0,00 & Muito inferior \\
\hline Stroop 2 - erros & $49,50(28,87)$ & 66,00 & Médio \\
\hline Stroop 3 - tempo & $0,00(0,00)$ & 0,00 & Muito inferior \\
\hline Stroop 3 - erros & $26,90(32,76)$ & 2,00 & Muito inferior \\
\hline TMT A & $0,30(0,80)$ & 0,00 & Muito inferior \\
\hline TMT B & $0,10(0,44)$ & 0,00 & Muito inferior \\
\hline $\begin{array}{l}\text { Span dígitos ordem inversa- WAIS } \\
\text { III }\end{array}$ & $51,05(34,70)$ & 62,00 & Médio \\
\hline WCST - respostas corretas & $86,75(18,11)$ & 96,50 & Superior \\
\hline WCST - categorias completadas & $38,8(47,10)$ & 7,00 & Inferior \\
\hline WCST - erros perseverativos & $39,85(30,73)$ & 38,00 & Médio \\
\hline $\begin{array}{l}\text { WCST - percentual de respostas } \\
\text { corretas }\end{array}$ & $46,65(40,32)$ & 44,00 & Médio \\
\hline $\begin{array}{l}\text { WCST - fracassos na manutenção de } \\
\text { contexto }\end{array}$ & $15,55(21,59)$ & 8,00 & Inferior \\
\hline
\end{tabular}

Quanto a análise por teste cognitivo, tem-se na tabela 3 a exposição dos desempenhos nos testes: Blocos de Corsi, Trail Making, nas formas A e B, bem como Dígitos para ordem inversa e MoCA. Neste ínterim tem-se que classificações muito inferiores foram encontradas para as medidas de pontuação total do Blocos de Corsi $(\mathrm{MED}=1)$, tal qual para TMT A $(\mathrm{MED}=0)$ e $\mathrm{B}(\mathrm{MED}=0)$, indicando prejuízos equivalentes a memória operacional visual, bem como rastreio visuomotor deficitário, respectivamente. Para a medida bloco span, do teste Corsi, a classificação do desempenho grupal equivale a média inferior, enquanto desempenhos mais satisfatórios foram encontrados em dígitos total $(\mathrm{MED}=56,2)$, bem como dígitos - ordem inversa $(\mathrm{MED}=62) \mathrm{com}$ classificações medianas.

Tabela 3. Desempenho cognitivo nos testes Blocos de Corsi, Trail Making (A e B), Dígitos- ordem inversa e MoCA.

\begin{tabular}{|c|c|c|c|c|c|c|c|c|c|c|c|c|c|c|}
\hline Sujeito & CS & $\mathrm{CL}$ & CT & $\mathrm{CL}$ & TA & $\overline{C L}$ & TB & $\overline{C L}$ & DT & $\mathrm{CL}$ & $\mathrm{DI}$ & $\overline{C L}$ & Mo & $\mathrm{CL}$ \\
\hline 1 & 0 & MTI & 0 & MTI & 0 & MTI & 0 & MTI & 25 & $\mathrm{MI}$ & 21 & $\mathrm{MI}$ & 22 & VI \\
\hline 2 & 5 & I & 0 & MTI & 0 & MTI & 0 & MTI & 25 & $\mathrm{MI}$ & 8 & I & 22 & VI \\
\hline 3 & 73 & M & 16 & $\mathrm{MI}$ & 0 & MTI & 0 & MTI & 90 & MS & 66 & M & 21 & VI \\
\hline 4 & 0 & MTI & 0 & MTI & 0 & MTI & 0 & MTI & 5 & 1 & 0 & MTI & 18 & VI \\
\hline 5 & 5 & I & 0 & MTI & 0 & MTI & 0 & MTI & 25 & $\mathrm{MI}$ & 21 & $\mathrm{MI}$ & 25 & VI \\
\hline 6 & 5 & I & 0 & MTI & 0 & MTI & 0 & MTI & 90 & MS & 10 & $\mathrm{MI}$ & 17 & VI \\
\hline 7 & 5 & I & 0 & MTI & 0 & MTI & 0 & MTI & 63 & $\mathrm{M}$ & 66 & $M$ & 20 & VI \\
\hline 8 & 46 & M & 12 & $\mathrm{MI}$ & 1 & MTI & 2 & MTI & 95 & $\mathrm{~S}$ & 98 & $S$ & 25 & VI \\
\hline 9 & 18 & $\mathrm{MI}$ & 4 & 1 & 2 & MTI & 0 & MTI & 90 & MS & 96 & $S$ & 29 & VI \\
\hline 10 & 5 & 1 & 0 & MTI & 0 & MTI & 0 & MTI & 75 & $M$ & 95 & $S$ & 25 & VI \\
\hline 11 & 46 & M & 7 & 1 & 0 & MTI & 0 & MTI & 25 & $\mathrm{MI}$ & 8 & I & 22 & VI \\
\hline 12 & 18 & $\mathrm{MI}$ & 2 & MTI & 0 & MTI & 0 & MTI & 16 & $\mathrm{MI}$ & 21 & $\mathrm{MI}$ & 20 & VI \\
\hline 13 & 18 & $\mathrm{MI}$ & 2 & MTI & 0 & MTI & 0 & MTI & 75 & $\mathrm{M}$ & 58 & $M$ & 27 & VI \\
\hline 14 & 46 & $M$ & 12 & $\mathrm{MI}$ & 3 & 1 & 0 & MTI & 84 & MS & 84 & MS & 25 & VI \\
\hline 15 & 46 & $M$ & 4 & I & 0 & MTI & 0 & MTI & 50 & $M$ & 58 & $M$ & 25 & $\mathrm{VI}$ \\
\hline 16 & 46 & $M$ & 7 & I & 0 & MTI & 0 & MTI & 75 & M & 84 & MS & 27 & VI \\
\hline 17 & 5 & 1 & 0 & MTI & 0 & MTI & 0 & MTI & 75 & $M$ & 73 & $M$ & 27 & VI \\
\hline 18 & 5 & 1 & 0 & MTI & 0 & MTI & 0 & MTI & 50 & $M$ & 73 & $M$ & 27 & $\mathrm{VI}$ \\
\hline 19 & 46 & $M$ & 7 & 1 & 0 & MTI & 0 & MTI & 50 & $M$ & 8 & 1 & 22 & VI \\
\hline 20 & 5 & 1 & 0 & MTI & 0 & MTI & 0 & MTI & 50 & $\mathrm{M}$ & 73 & $M$ & 27 & VI \\
\hline MED & 11,5 & & 1 & & 0 & & 0 & & 56,5 & & 62 & & 25 & \\
\hline DP & 22,2 & & 4,9 & & 0,8 & & 0,4 & & 28,5 & & 34,6 & & 3,3 & \\
\hline
\end{tabular}

$\mathrm{SU}=$ sujeitos, CS=Blocos de Corsi Span, CL=classificação, CT=Bloco de Corsi total, TA=Trail Making A, TB=Trail Making B, DT=Dígitos total, DI=Dígitos ordem inversa, Mo=MoCA. MTI=muito inferior, I=inferior, M=média, MI=média inferior, $S=$ superior, MED=mediana e $\mathrm{DP}=$ desvio padrão.

Acerca da frequência de déficits, fica evidenciado na Figura 1 expressivos déficits equivalentes as medidas obtidas pelos subtestes corsi - 
pontuação total, bem como TMT A e B.

Segundo Spreen e Strauss (1998) o resultado do teste Bloco de Corsi Span foi que $65 \%$ das pacientes tem déficits na função da memória operacional visual e no Bloco de Corsi Total foi que $100 \%$ tem déficits. No Trail Making A e B foram que $100 \%$ das pacientes estão com déficits na atenção seletiva e alternada, rastreamento visuomotor e flexibilidade cognitiva. Para o Teste Dígitos- ordem inversa foi que $40 \%$ das pacientes estão com déficits na memória operacional auditiva e no Dígitos Total foi que $30 \%$ delas tem déficits.

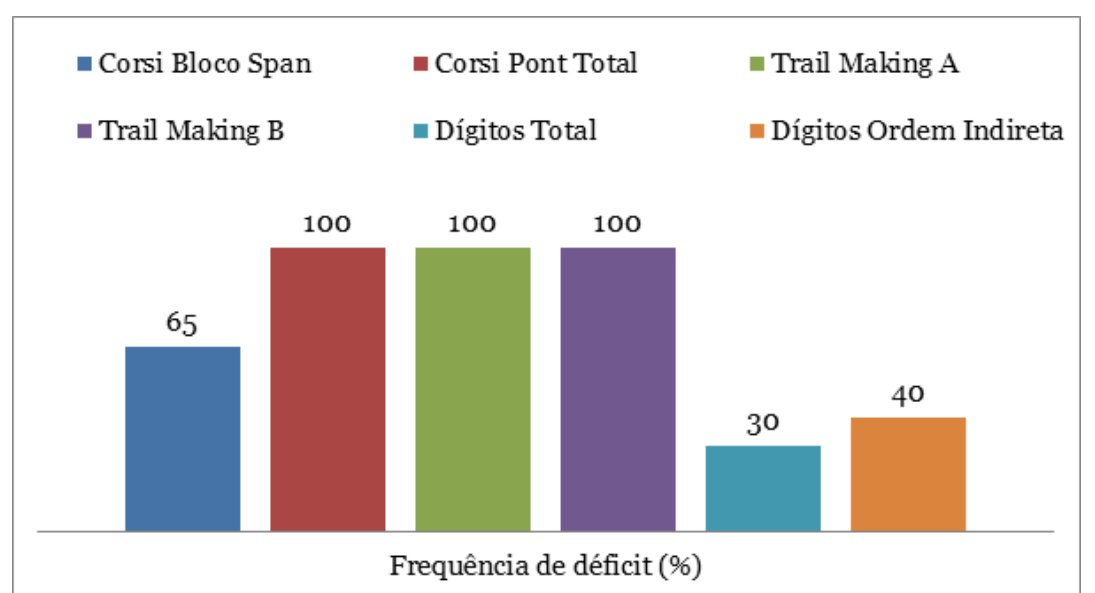

Figura 1. Frequência de déficits cognitivos demonstrados pelos testes Blocos de Corsi, TMT (A e B), Dígitos (pontuação total e ordem inversa)

Nas provas que mensuraram o controle inibitório, como domínio das funções executivas, os sujeitos apresentaram significativo prejuízo deste quesito, em função da expressiva latência associada para sustentar a atenção e controlar estímulos. A isso conferem os desempenhos muito inferiores encontrados nos subtestes Stroop 1,2 e $3(\mathrm{MED}=0$, em todos os subitens). Desempenhos melhores foram encontrados na configuração erros do Stroop Test, indicando que o baixo desempenho efetivamente estava correlacionado a latência da resposta e não ao comportamento impulsivo, no presente teste (Tabela 4).

Tabela 4. Desempenho cognitivo no Stroop Test

\begin{tabular}{c|cccccccccccc}
\hline Sujeito & ST1- & CL & ST1- & CL & ST2- & CL & ST2- & CL & ST3- & CL & ST3- & CL \\
& T & & E & & T & & E & & T & & E & \\
\hline 1 & 0 & MTI & 66 & M & 0 & MTI & 62 & M & 0 & MTI & 0 & MTI \\
2 & 0 & MTI & 0 & MTI & 0 & MTI & 0 & MTI & 0 & MTI & 2 & MTI \\
3 & 7 & I & 66 & M & 7 & I & 66 & M & 0 & MTI & 66 & M \\
4 & 0 & MTI & 66 & M & 0 & MTI & 2 & MTI & 0 & MTI & 66 & M \\
5 & 0 & MTI & 66 & M & 0 & MTI & 66 & M & 0 & MTI & 0 & MTI \\
6 & 0 & MTI & 66 & M & 0 & MTI & 0 & MTI & 0 & MTI & 66 & M \\
7 & 0 & MTI & 66 & M & 0 & MTI & 66 & M & 0 & MTI & 66 & M \\
8 & 1 & MTI & 66 & M & 0 & MTI & 66 & M & 0 & MTI & 66 & M \\
9 & 7 & I & 66 & M & 0 & MTI & 2 & MTI & 0 & MTI & 66 & M \\
10 & 1 & MTI & 66 & M & 4 & I & 66 & M & 0 & MTI & 66 & M \\
11 & 0 & MTI & 2 & MTI & 0 & MTI & 66 & M & 0 & MTI & 2 & MTI \\
12 & 0 & MTI & 66 & M & 0 & MTI & 66 & M & 0 & MTI & 0 & MTI \\
13 & 0 & MTI & 2 & MTI & 0 & MTI & 0 & MTI & 0 & MTI & 2 & MTI \\
14 & 0 & MTI & 66 & M & 0 & MTI & 66 & M & 0 & MTI & 0 & MTI \\
15 & 1 & MTI & 66 & M & 4 & I & 66 & M & 0 & MTI & 2 & MTI \\
16 & 0 & MTI & 66 & M & 0 & MTI & 66 & M & 0 & MTI & 66 & M \\
17 & 0 & MTI & 66 & M & 0 & MTI & 66 & M & 0 & MTI & 0 & MTI \\
18 & 0 & MTI & 66 & M & 0 & MTI & 66 & M & 0 & MTI & 0 & MTI \\
19 & 0 & MTI & 2 & MTI & 0 & MTI & 66 & M & 0 & MTI & 2 & MTI \\
20 & 0 & MTI & 66 & M & 0 & MTI & 66 & M & 0 & MTI & 0 & MTI \\
\hline MD & 0 & & 66 & & 0 & & 66 & & 0 & & 2 & \\
DP & 2,1 & & 26,4 & & 1,9 & & 28,8 & & 0 & & 32,7 & \\
\hline
\end{tabular}

ST1-T=Stroop 1 tempo, CL=classificação, ST1-E=Stroop 1 erros, ST2-T=Stroop 2 tempo, ST2-E=Stroop 2 erros, ST3-T=Stroop3 tempo, ST3-E=Stroop 3 erros, MTI=muito inferior, $\mathrm{I}=$ inferior, $\mathrm{M}=$ =média, $\mathrm{MD}=$ =mediana e $\mathrm{DP}=$ desvio padrão.

$\mathrm{Na}$ Figura 2, abaixo, ficam demonstradas as frequências de déficits apresentadas pela amostra deste estudo, indicando dificuldade expressiva em provas que requisitam controle inibitório, em detrimento do tempo de execução. Consta que $100 \%$ dos sujeitos apresentaram desempenhos abaixo da média em Stroop 1,2 e 3, quanto a variável tempo.

- Stroop Test 1 Tempo $\backsim$ Stroop Test 1 Erros $\quad$ Stroop Test 2 Tempo

- Stroop Test 2 Erros $\quad$ Stroop Test 3 Tempo $\backsim$ Stroop Test 3 Erros

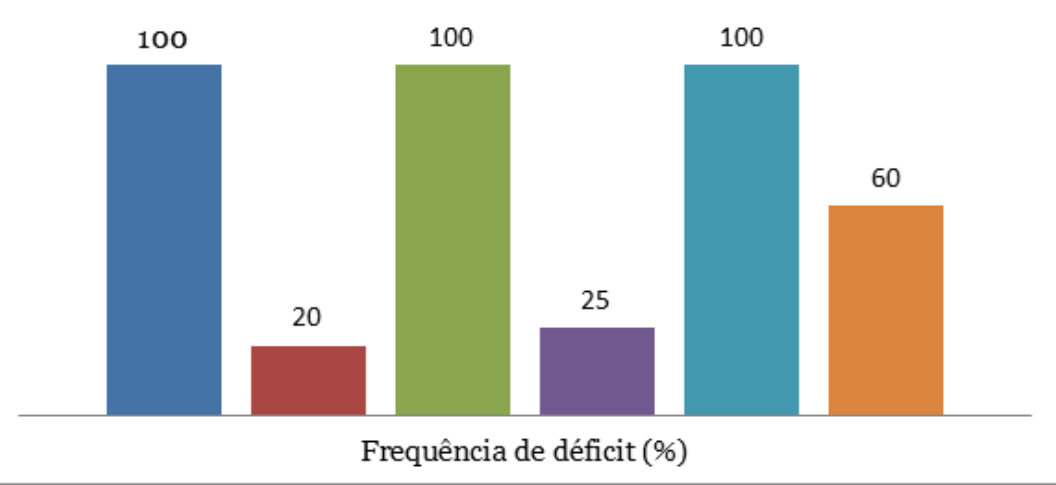

Figura 2. Frequência de déficits cognitivos demonstrados pelo Stroop Test

Inerente as medidas de flexibilidade cognitiva, categorização e impulsividade atencional, obtidas por meio do Teste Wisconsin, indicam: (1) classificação inferior para os subitens categorias completadas e fracasso na manutenção de contexto $(\mathrm{MED}=7)$; $(2)$ desempenhos medianos em erros perseverativos $(\mathrm{MED}=38)$ e resposta de nível conceitual $(\mathrm{MED}=44)$; e (3) desempenho superior para número de respostas corretas $(\mathrm{MED}=95)$. $\mathrm{O}$ presente dado reflete a expressiva dificuldade em manter o contexto e portanto adquirir categorias completas, a partir de feedbacks externos. Assim, embora façam acertos intermitentes, os erros por dificuldade em manutenção de categorias são frequentes, configurando inflexibilidade cognitiva (Tabela 5).

Tabela 5. Desempenho cognitivo no Teste Wisconsin de Classificação de Cartas

\begin{tabular}{c|cccccccccc}
\hline Sujeito & rc & cla & cc & cla & ep & cla & nc & cla & f & cla \\
\hline 1 & 46 & M & 0 & MTI & 0 & MTI & 0 & MTI & 34 & M \\
2 & 62 & M & 0 & MTI & 10 & MI & 7 & I & 34 & M \\
3 & 88 & MS & 1 & MTI & 16 & MI & 12 & MI & 34 & M \\
4 & 38 & M & 0 & MTI & 0 & MTI & 4 & MTI & 5 & I \\
5 & 99 & S & 62 & M & 54 & M & 84 & MS & 0 & MTI \\
6 & 84 & MS & 0 & MTI & 38 & M & 7 & I & 5 & I \\
7 & 99 & S & 99 & S & 76 & MS & 86 & MS & 69 & M \\
8 & 97 & S & 7 & I & 38 & M & 46 & M & 0 & MTI \\
9 & 99 & S & 99 & S & 79 & MS & 48 & S & 8 & I \\
10 & 96 & S & 7 & I & 21 & MI & 42 & M & 0 & MTI \\
11 & 82 & MS & 0 & MTI & 3 & MTI & 8 & I & 0 & MTI \\
12 & 99 & S & 99 & S & 90 & S & 96 & S & 8 & I \\
13 & 98 & S & 7 & I & 54 & M & 50 & M & 0 & MTI \\
14 & 84 & MS & 0 & MTI & 24 & MI & 12 & MI & 0 & MTI \\
15 & 86 & MS & 0 & MTI & 4 & MTI & 4 & MTI & 1 & MTI \\
16 & 82 & MS & 1 & MTI & 16 & MI & 7 & I & 8 & I \\
17 & 99 & S & 99 & S & 79 & MS & 93 & S & 69 & M \\
18 & 99 & S & 98 & S & 79 & MS & 92 & S & 14 & MI \\
19 & 99 & S & 99 & S & 58 & M & 93 & S & 8 & I \\
20 & 99 & S & 98 & S & 58 & M & 92 & S & 14 & MI \\
\hline MD & 95 & & 7 & & 38 & & 44 & & 8 & \\
DP & 18,1 & & 47 & & 30,7 & & 40,3 & & 21,5 & \\
\hline
\end{tabular}

$\mathrm{rc}=$ respostas corretas, cla $=$ classificação, $\mathrm{cc}=$ categorias completadas, ep $=$ erros perceverativos, $\mathrm{nc}=$ nível conceitual, $\mathrm{f}=$ fracassos, $\mathrm{MTI}=$ muito inferior, $\mathrm{I}=$ inferior, $\mathrm{MI}=$ médio inferior, $\mathrm{M}$ = médio, $\mathrm{MS}=$ médio superior e $\mathrm{S}=$ superior

A frequência de déficits cognitivos nos subitens do Wisconsin estão demonstrados na Figura 3, uma vez que, 55\% dos sujeitos apresentaram resultados abaixo da média para o subitem categorias completadas; $45 \%$, em erros perseverativos e respostas de nível conceitual; bem como, $75 \%$ da população 
deste estudo demonstrou respostas abaixo da média para a variável "fracassos na manutenção do contexto", do WCST (Figura 3).

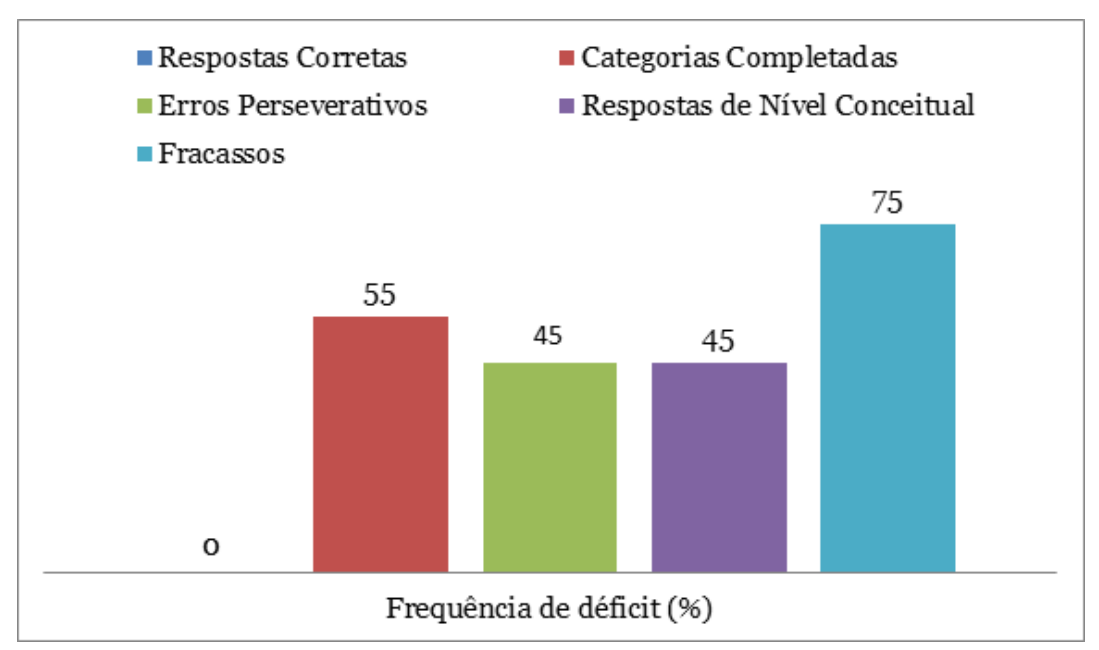

Figura 3. Frequência de déficits cognitivos demonstrados pelo Teste Wisconsin de Classificação de Cartas

Quando aplicados estudos correlação entre desempenho cognitivo e tempo de uso de substância química, embora não tenha sido encontrado um $p$ value $<0,05$, mas $\mathrm{p}=0,062(\mathrm{r}=0,425)$, deve ser considerada a significância clínica deste dado, uma vez que a análise quantiqualitativa acima exposta, indicou alta incidência de disfunção executiva nesta amostra. Do mais, correlações positivas também foram encontradas entre idade e tempo de dependência química, indicando a significativa prevalência da dependência química, ao longo da vida destes sujeitos $(\mathrm{p}=0014 ; \mathrm{r}=0,542)$.

\section{DISCUSSÃO}

Segundo Lezak et al. ${ }^{37}$, constitui objetivo da avaliação neuropsicológica, além da avaliação, o oferecimento de subsídios para a intervenção no comportamento humano, fazendo uma relação do funcionamento normal do sistema nervoso central, com o deficitário, por intermédio dos conhecimentos aplicados da neuropsicologia. Do mais, este instrumento analisa os distúrbios de comportamento e da cognição, causados por doenças, lesões, disfunções ou modificações experimentais. Deste modo, a presente configura-se uma importante ferramenta na detecção de prejuízos cognitivos apresentados por sujeitos em dependência química.

A hipótese de que déficits nas funções executivas dificultaria o tratamento e a recuperação de pacientes, fica evidente com os resultados desta pesquisa.

Conforme Bolla et al. ${ }^{32}$, o uso prolongado de cocaína pode causar anormalidades no funcionamento do córtex pré-frontal, que interfere no processo de tomada de decisão. Este aspecto ficou comprovado com os resultados do teste Wisconsin, especialmente com grande número de fracassos da maioria dos sujeitos, deixando claro que a decisão tomada foi errada e não conseguindo completar as categorias, tendo evidenciado um desempenho inferior. Sendo assim, pode-se inferir que, as pacientes estariam sujeitas, no decorrer do tratamento e pós-tratamento a tomadas de atitudes inadequadas, e como consequência, um retorno aos sintomas da doença.

Um estudo realizado por Cunha e Novaes ${ }^{28}$, com dependentes de cocaína, revelou um baixo desempenho em testes que avaliavam atenção, funções executivas, memória visual de longa duração e aprendizagem verbal, quando comparados a um grupo controle. Caso também comprovado nesta pesquisa, como o alto índice de respostas deficitárias no teste Bloco de Corsi, revelando baixo desempenho na memória visual. Déficits neste teste refletem prejuízos dos domínios de memória operacional visuoespacial.

Já o baixo nível de atenção ficou constatado no teste Wisconsin, pois propositalmente o teste é composto de muitos estímulos, o que pode direcionar o sujeito a dificuldades de sustentação atencional, prejudicando então as respostas equivalentes a manutenção de contexto, categorização e flexibilidade cognitiva. Também constatado nas observações feitas durante as atividades diárias das pacientes, quanto mais demorava uma atividade, mais dispersas elas ficavam, comprovando a dificuldade causada por este déficit.

Segundo Andrade et al. ${ }^{17}$ e Cunha e Novaes ${ }^{28}$, sujeitos dependentes tendem a apresentar importantes alterações cognitivas, principalmente nas funções mnemônicas, atencionais e executivas, como por exemplo, na memória de trabalho: controle e seleção de resposta (intenção): resolução de problemas e tomadas de decisão. O controle e a seleção de respostas dos sujeitos analisados estavam afetados conforme mostram os resultados do Stroop Test, onde a latência na execução do teste foi expressiva, configurando prejuízos das respostas. Do mais, a etapa 3 deste teste, refletiu um desempenho prejudicado, possivelmente em função da dificuldade da tarefa, onde $\mathrm{o}$ sujeito deveria recrutar alta capacidade atencional.

Ter controle inibitório para selecionar respostas é uma função extremamente importante para a dependente químico, pois ela necessita fazer escolhas diferentes e bem analisadas para evitar comportamentos que a induzem ao uso de drogas. Já a atenção, sugeri déficit, pois houve significativo prejuízo no tempo de execução no Trial Making Test, $\mathrm{A}$ e $\mathrm{B}$, e sem atenção ficariam propensas à recaídas $\mathrm{e}$ perda de todo trabalho árduo de seis meses de internação numa comunidade terapêutica, por exemplo.

Para Lorea et al. ${ }^{3}$, há prejuízos na fluidez verbal, como comprova o baixo rendimento no MoCA, especialmente no subteste de linguagem. Esta função é de real importância, pois para reinserção social ou familiar, elas necessitarão de uma 
comunicação adequada, quesito que deve ser estimulado dentro do tratamento, com consequente substituição das gírias, comumente utilizadas, mas inteligíveis pela sociedade.

Cumpriu-se o objetivo geral deste trabalho com os resultados obtidos nos testes neuropsicológicos, pois ficou constatada dificuldade nas funções executivas, como foi descrito anteriormente.

\section{CONCLUSÃO}

Os resultados permitiram concluir que:

- Mulheres com dependência química, pelo uso de cocaína e crack, apresentam disfunção executiva;

- Desempenhos mais rebaixados de funcionamento executivo, relacionados com os níveis atencionais, à memória operacional auditiva e visuoespacial, à flexibilidade cognitiva, planejamento, controle inibitório e rastreio visuomotor, foram encontrados em sujeitos do sexo feminino, com dependência a cocaína e ao crack;

- Relações clinicamente significativas foram encontradas entre tempo de uso e déficits do funcionamento executivo, indicando que quanto maior o tempo de dependência, mais expressiva a disfunção executiva.

\section{REFERÊNCIAS}

1. Organização Mundial da Saúde. Classificação de Transtornos Mentais e de Comportamento da CID10. Porto Alegre: Artes Médicas; 1993.

2. DSM-IV-TR. Manual Diagnóstico e Estatístico de Transtornos Mentais. 4 ed. (rev). Porto Alegre: Artmed; 2002. 880 p.

3. Negrete JC. Cocaine in the Coca-growing Countries of South America. 1992. Disponível em: http://www.ncbi.nlm.nih.gov/pubmed/1638920. Acessado em: 11/04/2014.

4. Maass VJ, Kirberg BA. La epidemia de cocaína: morbo en el norte de Chile. Rev Psiquiatria 1990;7:639-47.

5. Castaño G.A. Cocaínas Fumables em Latinoamérica. 2000. Disponível em: http://www.adicciones.es/files/castano.pdf. Acessado em: 18/05/2014.

6. Reinarman C, Levine HC. The Crack Attack: Politics and Media in the Crack Scale. 2003. Disponível em: http://www.corwin.com/upmdata/4006_Newman_Reader_

Chp_3_The_Crack_Attack_Final_Pdf.pdf.

Acessado em: 02/04/2014.

7. Ellenhorn MJ, Schonwald S, Ordog G, Wasserberger J. Ellenhorn's medical toxicology: diagnosis and treatment of human poisoning. $2^{\text {nd }}$ ed. Baltimore: Williams \& Wilkins; 1997.

8. OSAP - Office of Substance Abuse Prevention Crack Cocaine: A Challenge for Prevention.
Monograph-9. 1991. Disponível em: http://eric.ed.gov/?id=ED354444. Acessado em: 05/04/2014.

9. Procópio A. O Brasil no mundo das drogas. Petrópolis: Editora Vozes; 1999.

10. Uchoa MA. Crack: O Caminho das Pedras. São Paulo: Editora Ática. 1996. Disponível em: http://www.4shared.com/rar/6N8vF0Xf/7024629-

Marco-Antonio-Uchoa-Cr.html. Acessado em: 02/04/2014.

11. Dimenstein G. Repressão Amplia Limites da Cracolândia. São Paulo: Folha de São Paulo. 11/04/1999. Disponível em: http://www1.folha.uol.com.br/fsp/cotidian / ff11049914.htm. Acessado em: 18/05/2014. Disponível em: http://www.mocatest.org/ pdf_files/ instructions/ moca-instructions portuguese. pdf. Acessado em: 19/10/14.

12. Nappo SA, Galduróz JC, Raymundo M, Carlini EA. Changes in Cocaine Use as Viewed By Key Informants: A Qualitive Study Carried out in 1994 and 1999 in São Paulo, Brazil. 2001. Disponível em: http://translate.google.com.br/translate?hl=ptBR\&sl=en\&u=http://www.ncbi.nlm.nih.gov/pubm ed/11718317\&prev=/search. Acessado em: 05/04/2014.

13. Dunn J, Laranjeira R. Transitions in the Route of Cocaine Administration - Characteristics, Direction and Associated Variables. 1999. Disponível em: http://www.uniad.org.br/ desenvolvimento/images/stories/publicacoes/cocai na/cocaine\%20transitions.pdf. Acessado em: 18/05/2014.

14. Dias AC, Ribeiro M, Dunn J, Sesso R, Laranjeira R. Follw-up Study of Crack Cocaine Users: Situacion of the Patients After 2,5 and 12 Years. 2008. Diaponível em: http://www.uniad.org.br/ desenvolvimento/images/stories/pdf/Tese_Andrea _Dias_Parte_2.pdf. Acessado em: 18/05/2014.

15. Ribeiro LA, Sanchez ZM, Nappo SA. Estratégias desenvolvidas por usuários de crack para lidar com os riscos decorrentes do consumo da droga. J Bras Psiquiatr. 2010;59(3):210-8.

16. INPAD/UNIAD. II Levantamento Nacional de Álcool e Drogas - 2012. Disponível em: HTTP://www.inpad.org.br/ index.php?option $=$ com_content $\&$ view $=$ article $\& i d=106 \&$ Itemid $=9$ Acessado em: 20/04/2014.

17. Andrade VM, Santos FM, Bueno OFA. Neuropsicologia Hoje. São Paulo: Artes Médicas;2004.

18. Beatty J. Principles of behavioural neurosciences. Dubuque: Brown \& Benchmark; 1995.

19. Cosensa MR, Guerra LB. Neurociência e Educação: Como o Cérebro Aprende. Porto Alegre: Artmed; 2011. 
20. Fuentes D, Malloy-Diniz LF, Camargo CHP, Cozensa RM. \& Colaboradores. Neuropsicologia: Teoria e Prática. Porto Alegre: Artmed (2008).

21. Estévez-Gonzáles A, Garcia-Sánchez C, Barraquer-Boras LI. Los lóbulos frontales: el cérebro ejecutivo. Rev Neurol. 2000;31(6):56677.

22. Gall FJ. On the functions of the brain ando f each of its parts: with observations on the possibilities of determining the instincts, propensities and talents and head. Boston: Capen and Lyon; 1835.

23. Spurzheim JG. Phrenology on the doctrine of the mental phenomena. Boston: Capen and Lyon; 1833.

24. Feinberg TE, Farah ML. Behavioral neurology and neuropsychology. New York: McGraw-Hill; 1997.

25. Damásio AR. O Erro de Descartes: Emoção, Razão e o Cérebro Humano. São Paulo: Companhia das Letras; 1996.

26. Luria AR. Fundamentos de Neuropsicologia. São Paulo: Editora Universidade da Estado de São Paulo; 1981.

27. Figlie NB, Bordin S, Laranjeiras R. Aconselhamento em dependência química. São Paulo: Roca; 2004.

28. Cunha PJ, Novaes M. Avaliação Neurocognitiva no Abuso e Dependência do Álcool: Implicações para o Tratamento. Rev Bras Psiquiatr. 2004;26(supl. I):23-7.

29. Nassif SLS. Aspectos neuropsicológicos associados ao uso de cocaína. In: Andrade VM, Santos FH, Bueno OFA. Neuropsicologia hoje. São Paulo: Artes Médicas; 2004. p. 371-385.

30. Verdejo A, Orozco-Giménez C, Meersmans Sánchez-Jofré M, Aguilar de Arcos F, PérezGarcía M. The impact exerted by the severity of recreational drug abuse on the different components of the executive function.Rev Neurol. 2004;38(12):1109-16.

31. Lorea I, Fernández-Montalvo J, Tirapu-Ustárroz J. Landa N, López-Goñi JJ. [Neuropsychological performance in cocaine addiction: a critical review. Rev Neurol. 2010;51(7):412-26.

32. Bolla KI, Eldreth DA, London ED, Kiehl KA, Mouratidis M, Contoreggi C, et al. Orbitofrontal Cortex Dysfuncion in Abstinent Cocaine Abusers Performing a Decision-Making Task. Neuroimage. 2003;19(3):1085-94.

33. Fernàndez GG, Rodríguez OG, Villa RS. Neuropsicología y adicción a drogas. Papeles del Psicólogo. 2011.32(2):159-65.

34. Cunha JA. Manual do Teste Wisconsin de classificação de cartas: adaptação e padronização brasileira. São Paulo: Casa do Psicólogo; 2005.

35. Wechsler D. Wechsler adult intelligence scale. New York: The Psychological Corporation; 1981.
36. Milner B. Interhemispheric differences in the localization of psychological processes in man. $\mathrm{Br}$ Med Bull. 1971;27(3):272-7.

37. Lezak MD. Neuropsychological assessment.4rd ed. New York: Oxford University Press; 2004.

38. Trenerry MR, Crosson B, Deboe J, Leber WR. The stroop neuropsychological screening test. Odessa: Psychological Assessment Resources, 1989.

39. Camargo APA. Funções Neuropsicológicas Executivas Pós Acidente Vascular Encefálico Hemorrágico [dissertação]. Bauru: Universidade Estadual Paulista Júlio de Mesquita Filho UNESP; 2012.

40. Spreen O, Strauss EA Compendium of Neuropychological Tests: Administration, Norms, and Comentary. New York: Oxford University Press; 1998.

\section{CONFLITO DE INTERESSES}

Os autores declaram não haver conflitos de interesse.

\section{AUTOR PARA CORRESPONDÊNCIA}

Rui Mateus Joaquim

ruimateus@hotmail.com

Submetido em 21/01/2016 Aceito em 29/01/2016 